\title{
Liga de Puericultura
}

\author{
League childcare
}

\author{
Myris Satiko Shinzato ${ }^{1}$, Mariana Patrícia Dias de Campos Carvalho¹, Diego Campos \\ Gonzalez ${ }^{1}$, Ana Carolina Aguiar Kuhne', Ana Paula Scoleze Ferrer², \\ Ana Cecília Silveira Lins Sucupira ${ }^{3}$
}

Shinzato MS, Carvalho MPDC, Gonzalez DC, Kuhne ACA, Ferrer APS, Sucupira ACSL. Liga de Puericultura. / League childcare. Rev ed (São Paulo). 2013 jan.-mar.;92(1):57-61.

RESUMO: Introdução. A Liga de Puericultura, atualmente, conta com a participação de 22 acadêmicos do segundo, terceiro e quarto anos de graduação de Medicina, supervisionados por dois médicos assistentes. O objetivo principal da Liga é inserir os alunos gradualmente no atendimento de crianças. Entre os principais pontos abordados na Liga, destacam-se: a importância e estrutura da anamnese; os princípios do atendimento de puericultura, o desenvolvimento de competências no estabelecimento da relação médico-paciente, visando a construção de uma relação mais humanizada; o aperfeiçoamento das habilidades de comunicação, como a escuta e o olhar atento; o desenvolvimento de maior destreza para realizar o exame físico em crianças; além de enfatizar aspectos de promoção da saúde e prevenção de doenças. Objetivo. O trabalho tem como objetivo realizar uma análise crítica sobre o funcionamento da liga nos últimos anos, com base em um questionário respondido por 38 alunos participantes da Liga entre 2006 e 2012, com enfoque no motivo da participação nessa atividade extracurricular, principais pontos positivos e negativos, principais dificuldades encontradas durante as consultas, além de sugestões de como melhorar a dinâmica da Liga. Relato $d a$ Liga. A Liga realiza atendimentos ambulatoriais, uma vez por semana, sob supervisão de médicos assistentes, sendo que a cada quatro dias de atendimento são intercaladas aulas teóricas sobre as peculiaridades da consulta pediátrica e principais problemas de saúde da infância. A faixa etária dos pacientes atendidos pelos alunos varia de 1 mês a 10 anos de idade. Para participar da Liga o acadêmico deve fazer o curso introdutório e a prova, que acontecem no início de cada ano. A discussão envolve a relação médico-paciente específica do paciente pediátrico e seus responsáveis, o registro da consulta no prontuário da criança, além da definição de diagnósticos e condutas, com suas justificativas. A organização administrativa é feita pela diretoria da Liga composta por um presidente e três diretores que gerenciam as atividades semanais, em especial a agenda de pacientes e o curso introdutório. As atividades acontecem em dez salas do ambulatório da especialidade e em salas de aula. Entre os resultados apontados no questionário, o principal motivo de ingresso na Liga é conhecer melhor a especialidade pediátrica, os principais pontos positivos são o aprendizado de puericultura e a longitudinalidade no acompanhamento do paciente; os negativos, poucos discutidores e falta de materiais, como o otoscópio; e a principal dificuldade é realizar a otoscopia e a oroscopia no exame físico. Como meta pretende-se que os objetivos da Liga sejam alcançados e as sugestões apresentadas, como aumentar o número de discutidores, sejam concretizadas.

DESCRITORES: Cuidado da criança; Pediatria. KEYWORDS: Child care; Pediatrics.

$2^{\circ}$ lugar Prêmio Painéis Área Liga Acadêmica - XXXI Congresso Médico Universitário da FMUSP - COMU 2012.

${ }^{1}$ Acadêmico(a) de Medicina da Faculdade de Medicina da Universidade de São Paulo - FMUSP.

2. Mestre em Pediatria pela Faculdade de Medicina da Universidade de São Paulo - FMUSP, Orientadora e Médica Assistente do Instituto da Criança do Hospital das Clínicas da Faculdade de Medicina da Universidade de São Paulo - FMUSP.

${ }^{3}$ Mestre em Medicina Preventiva pela Faculdade de Medicina da Universidade de São Paulo - FMUSP, Doutora em Pediatria pela

FMUSP, Orientadora e Professora Colaboradora do Departamento de Pediatria da FMUSP.

Endereço para correspondência: Universidade de São Paulo, Hospital das Clínicas da FMUSP, Instituto da Criança. Av. Enéas de Carvalho Aguiar, 647- São Paulo, SP. CEP: 05403-000. e-mail: myris.shinzato@gmail.com 


\section{INTRODUÇÃO}

\section{Histórico}

A Liga de Puericultura da Faculdade de Medicina da Universidade de São Paulo foi fundada em 1956, pelo Prof. Pedro de Alcântara Machado, sendo a terceira Liga criada na faculdade. Até 1971, quando teve início o estágio no quarto ano, em um Centro de Saúde do município, a Liga era o único contato que os alunos de graduação tinham com a Puericultura. Em 1976, com a inauguração do Instituto da Criança, a liga passou a funcionar às $3^{\mathrm{a}}$ feiras, com atendimento das 16 às $18 \mathrm{~h}$, seguindo-se uma aula teórica, com a participação de alunos do $4^{\circ}$ ao $6^{\circ}$ ano. Em 2003, a Liga passou a ser considerada disciplina optativa "Introdução à puericultura e à pediatria" para os alunos de $3^{\circ}$ e $4^{\circ}$ ano. Em 2008, iniciou-se uma experiência com a participação de alunos do $2^{\circ}$ ano. Na década passada, em função da baixa procura pela especialidade pediátrica, a Liga teve pouca procura pelos alunos, tendo em 2007, menos de 10 alunos. Vale ressaltar que esse foi um momento de aparecimento de muitas Ligas na Faculdade. Nos últimos 3 anos houve um grande aumento do número de alunos participantes. Atualmente, assim como todas as outras Ligas da Faculdade, ela pode ser considerada como a optativa Iniciação Profissional à Prática Médica.

Em 2012, a Liga conta com a participação de 22 acadêmicos do $2^{\circ}, 3^{\circ}$ e $4^{\circ}$ anos de graduação de Medicina, supervisionados por dois médicos assistentes.

\section{RELATO DA LIGA}

\section{Objetivos da Liga}

O objetivo principal da Liga é inserir os alunos gradualmente no atendimento de crianças. Os objetivos específicos de aprendizado na Liga são:

$>$ A importância e estrutura da anamnese;

$>$ Os princípios do atendimento de puericultura;

$>\mathrm{O}$ desenvolvimento de competências para o estabelecimento da relação médico-paciente, visando à construção de uma relação mais humanizada;

$>\mathrm{O}$ aperfeiçoamento das habilidades de comunicação;

$>\mathrm{O}$ desenvolvimento de maior destreza para realizar o exame físico em crianças;

$>$ Os aspectos de promoção da saúde e prevenção de doenças.

\section{Funcionamento}

A Liga realiza atendimentos ambulatoriais em dez salas do ambulatório do ICr HC-FMUSP, uma vez por semana, sob supervisão de médicos assistentes. A cada quatro dias de atendimento são intercaladas aulas teóricas sobre as peculiaridades da consulta pediátrica e principais problemas de saúde da infância. Como o atendimento é centrado na puericultura, procura-se matricular crianças nos primeiros meses de vida. Entretanto, no seguimento das crianças que é feito até os 10 anos de idade, o aluno aprende a atender os principais problemas da faixa pediátrica.

Em cada sala, 1 a 2 alunos atendem no máximo dois pacientes por dia. Os alunos são donos das agendas, acompanhando os pacientes durante todo o ano, possibilitando o vínculo com a criança e a família e a observação do desenvolvimento da criança.

Todos os alunos têm de discutir todos os casos atendidos. Após completar a anamnese e o exame físico e colocarem as suspeitas diagnósticas o aluno vai discutir o caso com o médico assistente. Nessa discussão, a ênfase é dada à relação médico-paciente específica do paciente pediátrico e seus responsáveis, ao registro da consulta, no prontuário da criança, além da definição de diagnósticos e condutas, com suas justificativas.

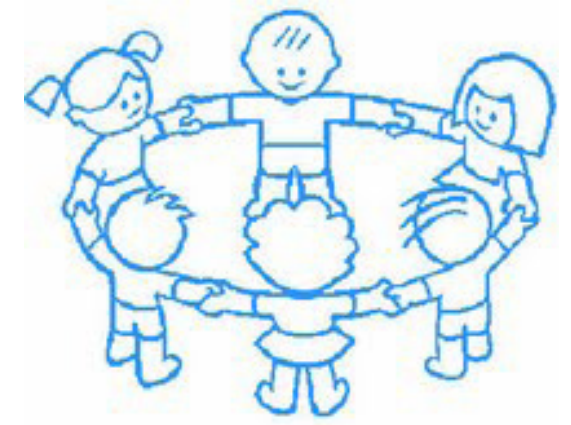

\section{Membros Acadêmicos}

Para participar da Liga o acadêmico deve fazer o curso introdutório e a prova, que acontecem no início de cada ano. Podem participar alunos do $2^{\circ}, 3^{\circ}$ e $4^{\circ}$ anos de medicina.

Com os alunos dos $2^{\circ}$ ano, há uma inserção gradual no atendimento. Primeiramente participam de discussões teóricas sobre a importância da anamnese e sua estrutura. Depois realizam uma conversa informal com a família da criança, enquanto os pacientes esperam para serem atendidos. Posteriormente acompanham consultas realizadas por alunos mais graduados. E por fim realizam consulta sob supervisão para poder futuramente atender em uma sala.

Os alunos do $3^{\circ}$ e $4^{\circ}$ ano atendem sozinhos os pacientes nas salas, e posteriormente discutem com os médicos, que entram no consultório antes da definição das condutas para o caso discutido.

\section{Organização administrativa}

A organização administrativa é feita pela diretoria 
da Liga composta por um presidente e três diretores que gerenciam as atividades semanais, em especial a agenda de pacientes, o curso introdutório, o controle da presença dos alunos.

\section{Pacientes}

A faixa etária dos pacientes atendidos pelos alunos varia de 1 mês a 10 anos de idade. A maior parte são filhos e netos de funcionários do HC-FMUSP, e crianças encaminhadas da Liga do Pré-Natal.

\section{Análise sobre a Liga}

Foi realizada uma pesquisa com membros e exmembros acadêmicos da Liga de Puericultura a fim de obter análise crítica sobre o funcionamento da liga nos últimos anos.

Com base no questionário respondido por 38 alunos participantes da Liga entre 2006 e 2012, foi possível analisar os motivos da participação nessa atividade extracurricular, principais pontos positivos e negativos, principais dificuldades encontradas durante as consultas, além de sugestões de como melhorar a dinâmica da Liga. Entre esses alunos, $63 \%$ consideraram como opção realizar
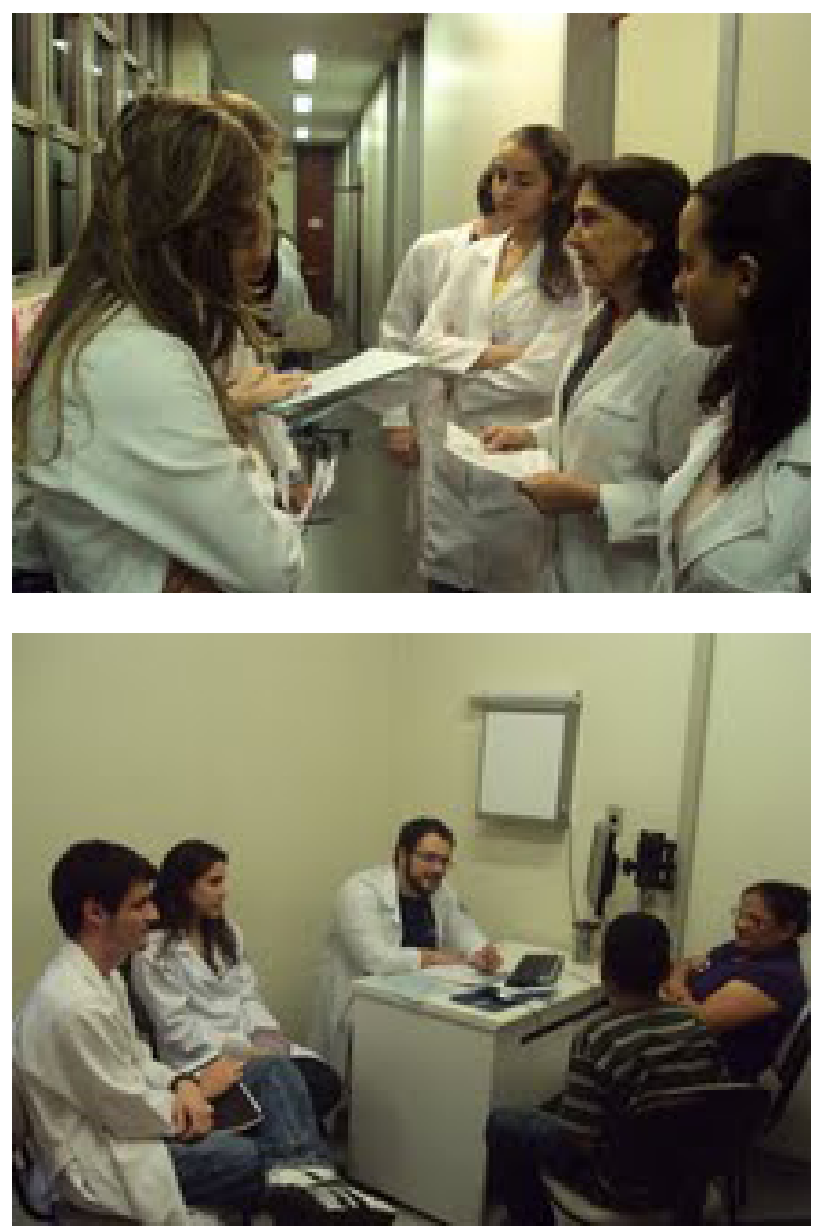

residência de Pediatria no futuro.

\section{RESULTADOS}

Entre os resultados apontados no questionário, o principal motivo de ingresso na Liga é conhecer melhor a especialidade pediátrica. Os principais pontos positivos são o aprendizado de puericultura e a longitudinalidade no acompanhamento do paciente; entre os negativos, poucos discutidores e falta de materiais para o atendimento, como o otoscópio. A principal dificuldade e fazer a otoscopia e a oroscopia no exame físico.

\section{PERSPECTIVAS FUTURAS}

Realizar a filmagem das consultas feitas pelos alunos com feedback.

Apresentação de consultas feitas pelos professores para discussão.

Aumentar a participação dos residentes da pediatria na supervisão dos alunos.

Desenvolvimento de trabalhos de iniciação científica - pesquisa tipo coorte com os pacientes da Liga para avaliar o desenvolvimento de crianças.
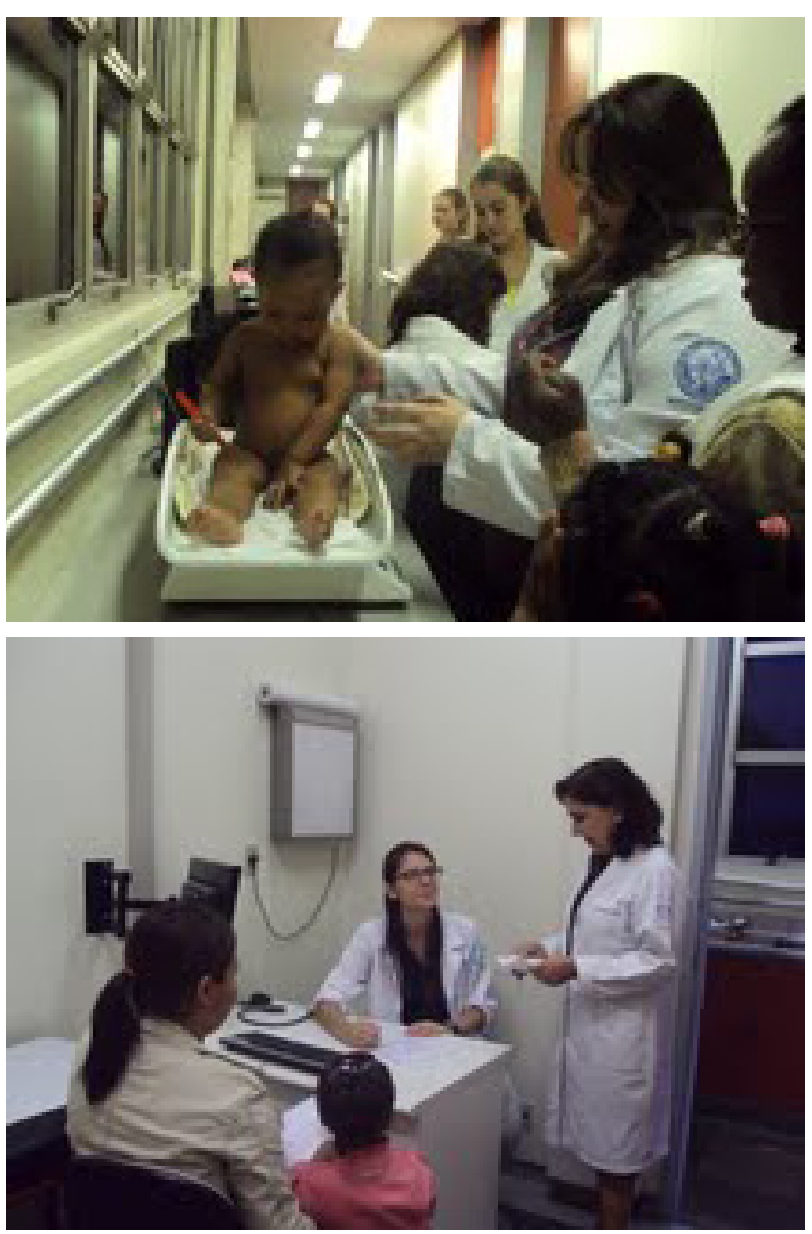


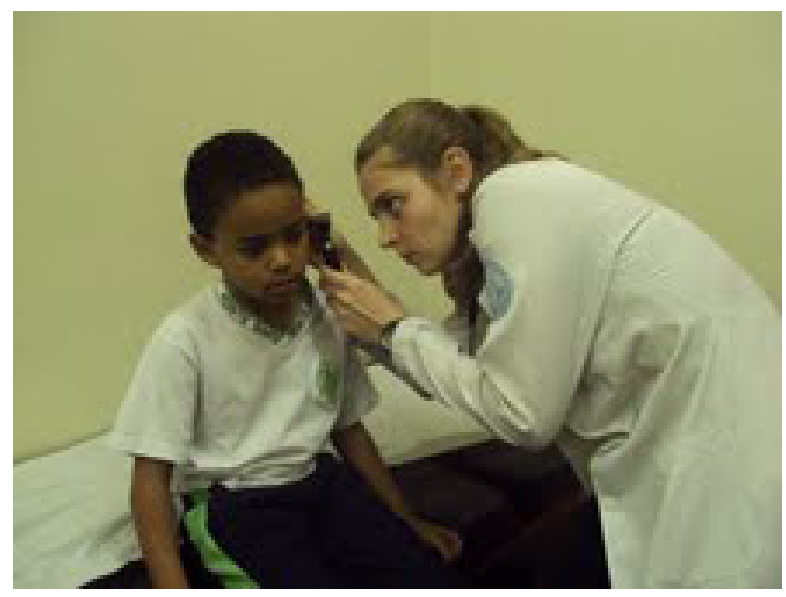

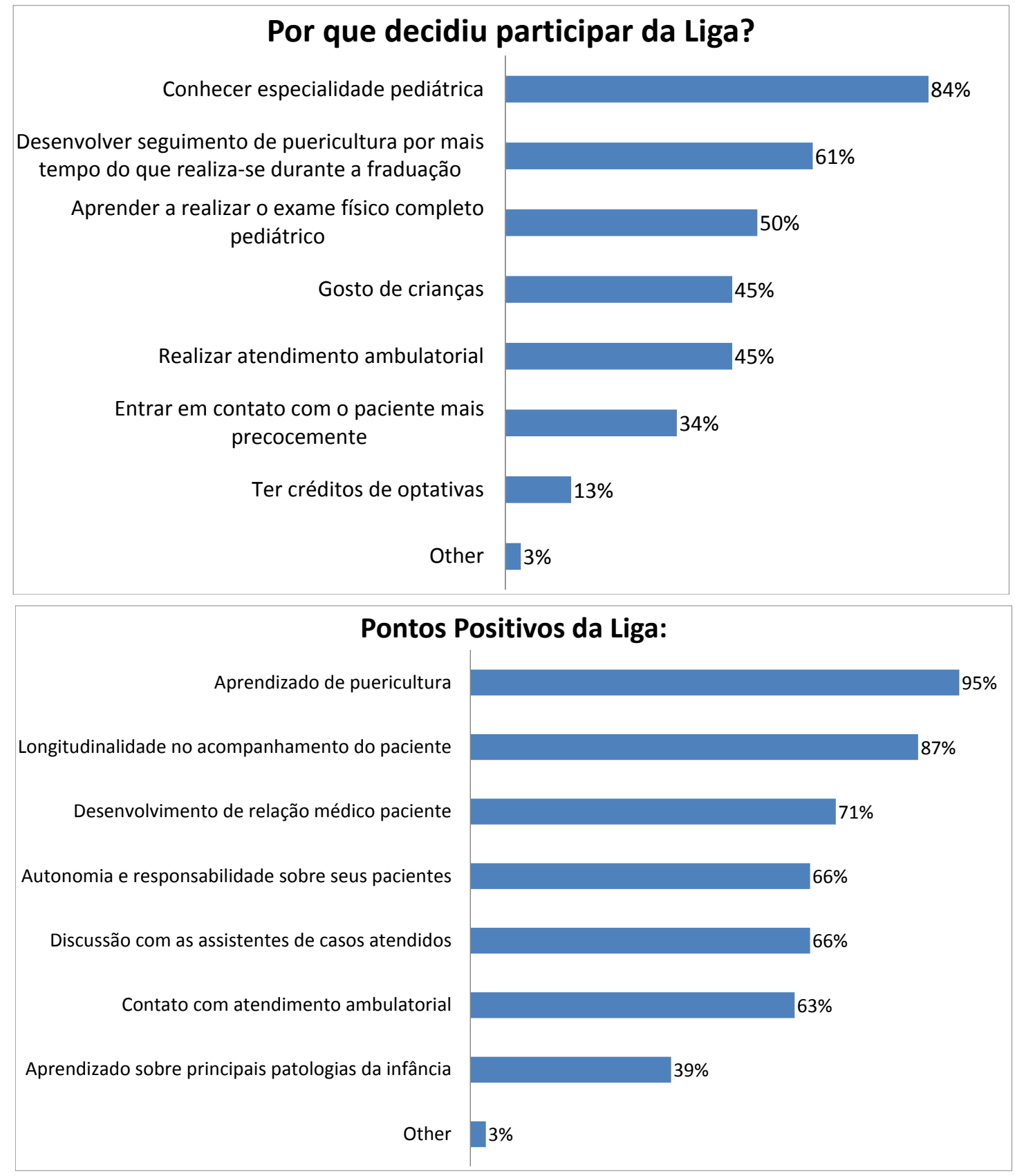



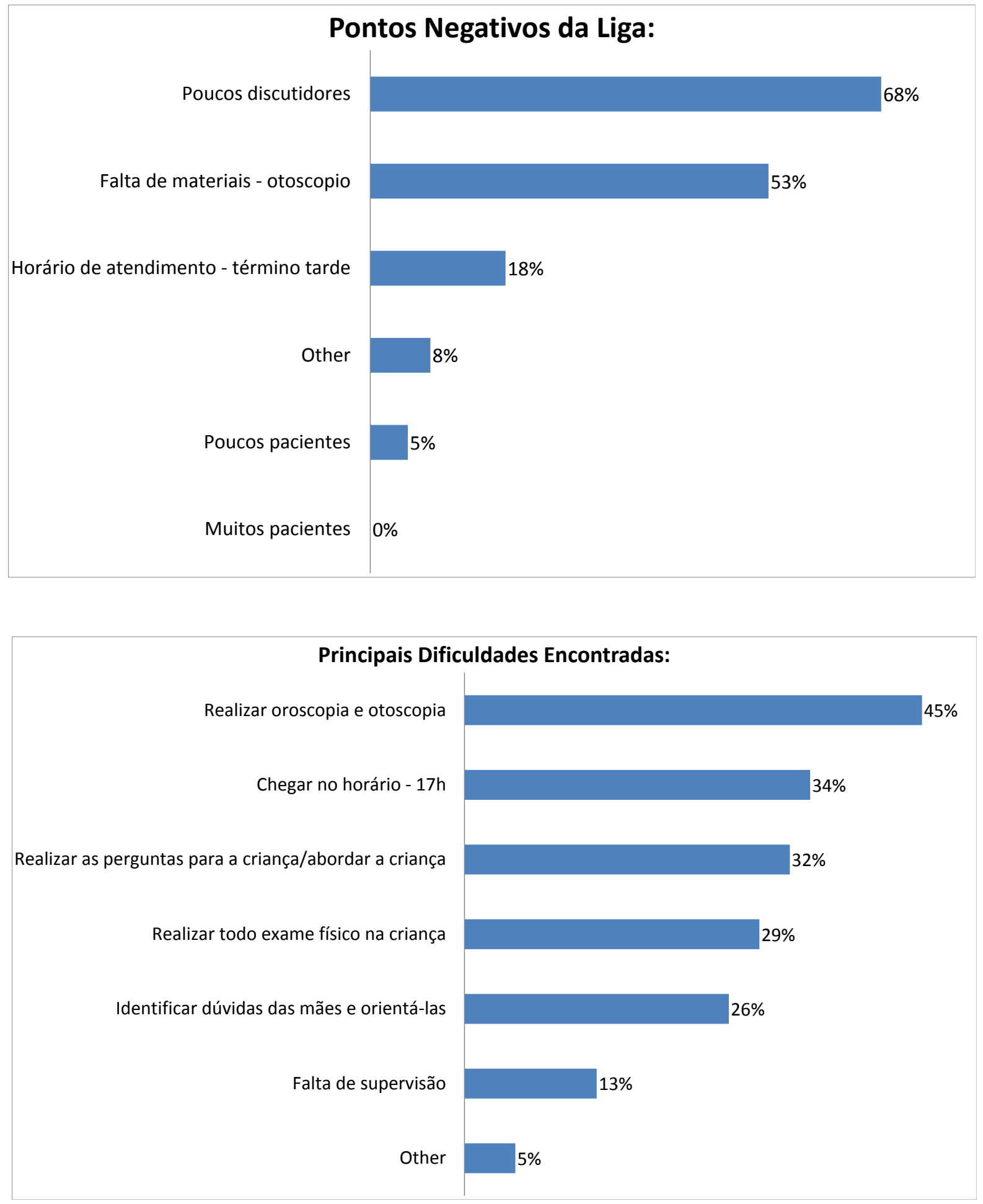